\title{
0198 CONSTRUCTION OF “POTENTIAL RISK ESTIMATION DROWNING INDEX FOR CHILDREN" (PREDIC): THE DROWNING RISK PREDICTION INDEX USING MATLAB SURVEILLANCE DATA, BANGLADESH
}

N N Borse, * A A Hyder, D Bishai Correspondence: CDC, 4700 Buford Hwy NE MS F-62 Atlanta, GA 30341, USA

\subsection{6/ip.2010.029215.198}

Aim The main objective of this paper was to create and evaluate a drowning risk prediction index. Drowning risk prediction index was constructed based on a conceptual framework to understand the determinants of childhood drowning.

Methodology A literature review was carried out to list all the risk factors identified for childhood drowning in Bangladesh. Newacheck model for special healthcare needs for children was adopted, to develop the conceptual framework for determinants of childhood drowning in Matlab, Bangladesh. This framework was applied to construct childhood drowning risk index for Matlab called Potential Risk Estimation Drowning Index for Children (PREDIC). Finally, PREDIC Index was applied childhood drowning cases and compared with control group from a cross-sectional data for children living in Matlab. Risk factors pertaining to childhood drowning were categorised in four key domains: child, mother/parent, household and environment factors.

Results For the purpose of this paper, PREDIC index was applied on 624 children living in Matlab and 302 drowned children in the $0-4$ years age group. The mean PREDIC score for the drowned children was significantly higher than those living children in Matlab. The ROC curve for PREDIC showed that index is a fairly good at drowning risk prediction.

Conclusion PREDIC risk index is created to estimate occurrence of childhood near-drowning event and not for drowning death. Index score construction was scientifically valid; index is relatively complete, fairly accurate, and very practical. PREDIC should be further tested for its feasibility and effectiveness in Matlab, Bangladesh. 